\title{
IEEE 802.11ah-Enabled Internet-of-Drone Architecture
}

\author{
Mehbub Alam, Student Member, IEEE, Nurzaman Ahmed, Member, IEEE, Rakesh Matam, Member, IEEE \\ Ferdous A. Barbhuiya, Member, IEEE
}

\begin{abstract}
IEEE 802.11ah is considered as a promising and scalable solution for connecting a large number of drones. With the support of sub-1GHz channel bands, relay, and group-based channel access mechanism, 802.11ah can help drone stations for collecting commands, sending data, and processing response to the control room. However, achieving required throughput and latency is still challenging due to high mobility and dynamic relaying requirement. This article proposes an IEEE 802.11ahbased Internet-of-Drones (IoD) architecture for surveillance and remote control. Our scheme predicts the location of a drone and places the required association and channel access configuration to a relay node dynamically. Moreover, the current mode of a drone station is switched to relay from station as per the location. The relay nodes use transmission opportunity (TXOP) with implicit acknowledgement to increase the efficiency of the network. The performance analysis shows significant improvement in terms of throughput and latency.
\end{abstract}

Index Terms-Internet-of-Drone, UAV, IEEE 802.11ah, RAW, Gauss-Markov Model, Drone Swarm.

\section{INTRODUCTION}

$\mathbf{T}$ HE emerging technology, Unmanned Aerial Vehicles (UAVs) network harnessed application spaces in services to public and military such as smart transport monitoring, crop management, food delivery, border area movement monitoring, forest surveillance, rescue and evacuation operations in the event of any disaster [1]. Internet-of-Drones (IoD) connects such networks to the Internet for improved decision making and control. Due to any unpredictable events such as floods, earthquakes, cyclones, terrorist attacks, and fire incidents, the adoption of IoD is advantageous. Moreover, IoD can be used to supply medical aids when it is not possible for a human being to reach the area physically. However, issues arise when the drones operate in the open, which needs a movement facility with more extensive geographic coverage while maintaining a healthy data rate for the drones.

IEEE 802.11ah has enormous potential in terms of covering larger geographical area and associating a large number of devices in the network. For achieving better data-rate (150Kbps78Mbps), 802.11ah standard uses different sub-1GHz channel bands, and Modulation and Coding Schemes (MCSs) with low power consumption [2]. The channel access feature of 802.11ah facilitates the stations to choose a suitable channel

M. Alam, R. Matam, and F. A. Barbhuiya are with the Department of Computer Science and Engineering, Indian Institute of Information Technology Guwahati, India, e-mail: mehbub@iiitg.ac.in, rakesh@iiitg.ac.in, ferdous@iiitg.ac.in

N. Ahmed is with the Department of Computer Science and Engineering, Indian Institute of Technology Kharagpur, India, e-mail: nurzaman@cse.iitkgp.ac.in

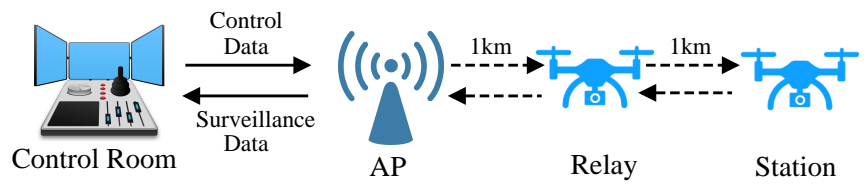

Fig. 1: Overview of the proposed architecture

for uniform usage. Also, with multi-hops or relay, it brings advantage to cover more areas, provision to a maximum of 2 hops and each hop can provide service to $1 \mathrm{~km}$ range. Managing such an extensive network dynamically from a remote place is well performed by this technology. Moreover, managing such a network using different MCSs to control the traffic congestion is a significant attribute of this technology [3]. IEEE 802.11ah supports a large number of devices at the sensing/actuation field and backbone networks. Therefore, complexity and heterogeneity in terms of networks, protocols, and packet formats have been automatically reduced. Managing such a network becomes easy with its growing size.

\section{A. Motivations}

IoD has an enormous number of applications in the domain of next-generation networking. An example scenario can be a set of drones moving in an area to identify any destruction caused by an earthquake. The drones are constantly on the move from one place to another while being interconnected with other drones are expected to maintain a smooth data rate, and connectivity while covering a wide area. It is challenging to tackle these two problems of maintaining a higher data rate and large area coverage in traditional drone network architecture. Mobile Ad-hoc Networks (MANET) uses a Random Walk mobility model, and Vehicular Ad hoc Network (VANET) uses the Street Random Walk or Manhattan mobility model. However, UAVs are not suitable for such mobilityaware behaviour. Unlike MANET and VANET, swarms in UAVs move in a three-dimensional pattern. The UAV networks change its topology quite frequently, and handling this issue is a challenge. It is needed to hold information such as the relative position of each drone, their energy state, communication link states with other drones, malfunctioning reports etc. Unlike VANET, the energy consumption in a drone network is more critical. In the vehicular scenario, we can recharge from the vehicle's battery, whereas we need to take down the drone for this purpose in a UAV network.

For the drone network application in disaster management, it is expected that the network is split up multiple times; existing 
technologies are not enough to handle this connectivity issue. A fully autonomous and relay-based drone network needs robust inter-drone network connectivity with neighbouring drones to keep the network alive. To deal with the link failure or device failure, the existing solutions such as WiFi, IEEE 802.15.4 are not sufficient. IEEE 802.11ah is a new technology, it considers group-based channel access, hierarchical addressing, multi-hop transmission opportunity (TXOP) etc. features which makes it suitable for IoD. However, a proper architecture has to be defined to overcome the challenges such as scalability, high mobility, longer coverage, and required uplink bandwidth. The drone network requires Medium Access Control (MAC) and network layer changes for self-organizing, delay-tolerant, flexible services, etc. Due to the multi-band and multi-MCSs characteristics of the 802.11ah standard, we can apply this to many multi-user scenarios having different Quality of Service (QoS) requirements for application such as video surveillance using a swarm of UAVs.

\section{B. Contribution}

We propose a network architecture for IoD, based on IEEE 802.11ah to connect large number of drones with multi-hop facilities. The overview of the proposed architecture is shown in Fig. 1. The proposed scheme predicts the location of the drones using the Gauss-Markov method. As per the location of the drone, the association and channel access configurations such as (i) Address Identifier (AID), (ii) Restricted Access Window (RAW), and (iii) Traffic Indication Map (TIM), are delivered to a new relay. A station is switched to relay mode if it comes between the AP and station in real-time. The surveillance video data are efficiently transferred to the AP node via relays using transmission opportunity (TXOP) operation.

\section{RELATED WORKS}

The existing related literature is mainly categorised as relay support, mobility and UAV used in 802.11ah. In our extensive searches, we found very few work has been done on the IoD application platform based on IEEE 802.11ah. Some of the important contributions are as follows:

\section{A. Relay support in 802.11ah}

The communication technology, IEEE 802.11ah comes with a promising feature of its ability to impose multi-hop operation with relays or mesh networking to extend coverage. One single Access Point (AP) can cover a range of $1 \mathrm{~km}$ area, and 8091 number of devices can be associated, and maximum relay node can be one. The relay facility the devices to use high data rates and TXOP sharing. Kocan et al. [4] examined the increased range possibility using relays in IEEE 802.11ah and implemented the communication between AP and end-user device with half-duplex decode-and-forward relay station. In [5] Kumar et al. presented and implemented a hierarchical MAC scheme for efficient management and deployment of relay nodes in using NS-3 simulator. The authors claimed of achieving more throughput and less energy consumption by covering extended areas using multi-channel relays. Their simulation result provides 18 times higher throughput in their scheme than other single-channel IEEE 802.11ah. Rao et al. [6] provided a detailed analysis on the dual-hop and the evidence of feasibility to use this claimed feature of IEEE 802.11ah. In context to the practical application scenario of a relay-based network system using 802.11ah technology, Ahmed et al. [7] presented a relay-based scheme elaborated how network bandwidth to the devices is allocated by these relay nodes while using multiple MCSs choices in different relay groups. However, the authors did not explain how the relay nodes are selected and also about their energy consumption.

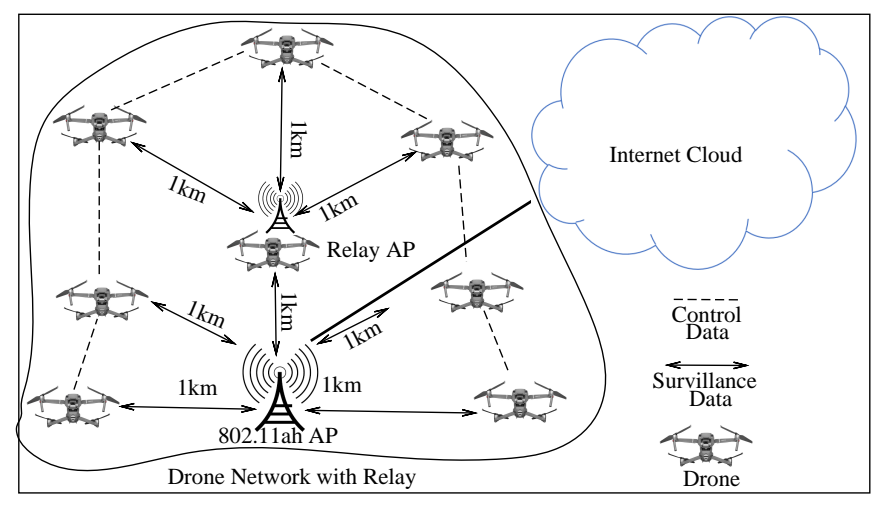

Fig. 2: Proposed 802.11ah based Internet of Drone Network Architecture

\section{B. Mobility in 802.11ah}

A very large number of devices can be associated with IEEE 802.11ah, which also facilitate the communication in IoT network among the mobile devices along with static deployed devices. Servicing smooth mobility features in large network architecture is a crucial QoS requirement for IoT. Most of the existing researches on mobility-aware wired or wireless technology are limited to a two-dimensional mobility strategy. In the literature, we find the Random Waypoint model, which describes as: to reach a destination, in the beginning, the device moves with random speed toward random destinations and then after pausing many times; finally, it starts moving towards the original destination with its coordinate. A few more mobility models exist in literature to represent real scenarios in simulation, such as Random Walk, Manhattan and Gauss-Markov mobility models. Muktiarto et al. [8] presented the details about the performance analysis of mobility using above mentioned three mobility models. The authors claim that the Random Waypoint mobility model performs better over the two other models in the NS-3 simulation environment. Similarly, the authors in [9] experimented with the Random Waypoint mobility model for performance analysis in 802.11ah of RAW and device speed.

\section{UAV using 802.11ah}

The UAVs play a vital role by providing assistance via air to the affected people in a disaster time. In the process, 
the drone network needs to serve with the mobile drones in the affected area. In such a scenario, the affected area is undoubtedly large; hence we find 802.11ah with relay APs is the most suitable option for both the challenges, i.e., mobility and large area coverage. Due to the advantage of multiple hops in 802.11ah technology to expand coverage area using a relay, Ali et al. [10] proposed architecture for UAV control network. In [11], the authors presented clustering technique based networking and security architecture for disaster surveillance UAV system for IEEE 802.11ah. This security scheme demands providing high accuracy and availability of the collected information received through drones and applied redundancy techniques. To get a larger area and high throughput in a drone network of surveillance using UAV swarm, Khan et al. [12] proposed PHY layer framework for communication between UAV to UAV and UAV to ground control room. In experimental results, it is observed that better performance for lower MCSs than higher MCSs.

Synthesis: The existing solutions are not suitable for connecting a large number of drones covering larger distances. They fail to provide dynamic data forwarding and efficient traffic scheduling considering both high volume video traffic and downlink control traffic.

\section{The Proposed IoD Architecture}

This article proposes an IEEE 802.11ah-based network and communication architecture for IoD. Fig. 2 shows the proposed architecture, where drone stations are mobile within a kilometer range of the ground AP. When a station goes beyond the $1 \mathrm{~km}$ range of the associated ground AP or any other available AP's, the associated AP takes the privilege to make one of the surrounding drone stations of the out of range drone as a relay station. Further, such switch operation is done with the motivation to increase its range through the relay station. The proposed solution considers a set of 802.11ah-based drone nodes which have the working options- relay mode and station mode. Based on the location and reference position of a node, the modes can be switched from relay to station and viceversa. All the nodes are centrally connected to one, or a set of 802.11ah based AP nodes, placed in the ground. Due to the association and handover delay between one AP to another AP and switching the station modes between AP and stations, the communication delay and disconnectivity may occur. To solve this problem, our approach predicts the location of the drone using Gauss-Markov's based approach. Thus, AP dynamically handsover the required association and access configuration of the respective mobile nodes to the AP or relay based on the predicted location. So the proposed scheme considers IoD traffic such as up-link multimedia data and download control traffic while scheduling RAW for a node.

\section{A. Prediction of location of drones}

For the IoD network paradigm, the drones are constantly moving and are connected to one of the APs to communicate and exchange information with the backbone network. Hence, the APs creates and manages network configurations for the

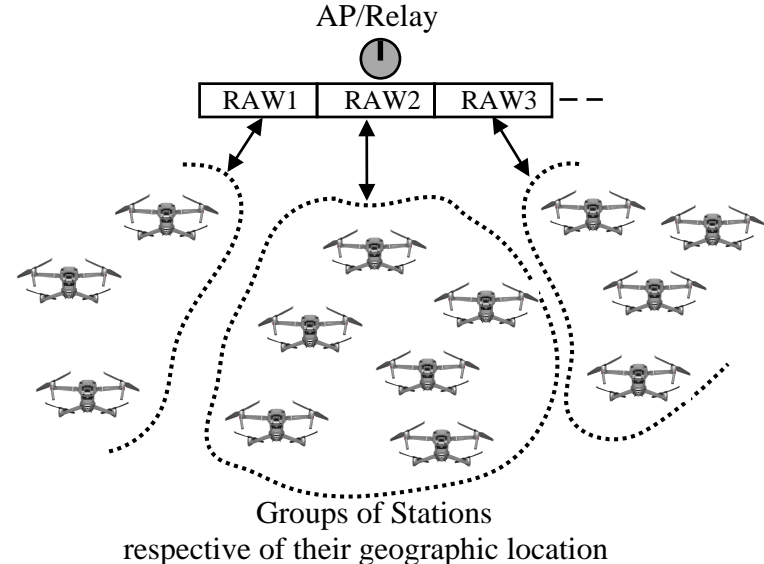

Fig. 3: Group of stations are placed with respect to their geographic location

drones. In a real world environment, as the location of drones changes frequently, the APs dynamically updates the table periodically for the new locations. To reduce this delay, we should predict the future location based on the mobility pattern of a drone to start the hand-off proactively. A functional drone speed and direction do not change quite frequently, but it gradually changes its direction. Assuming the drones movement follows Gaussian distribution and exponential distribution.

It is challenging to predict locations in the wireless network scenario; we use Gauss-Markov prediction [13] algorithm for mobile wireless systems. There exist a few more algorithms in the literature, such as Bayesian Network, Curve Fitting and Neural Network are introduced for designing predicting methods [14]. Using the Gauss-Markov mobility prediction model, we find the future location based on the previously visited location by the drones.

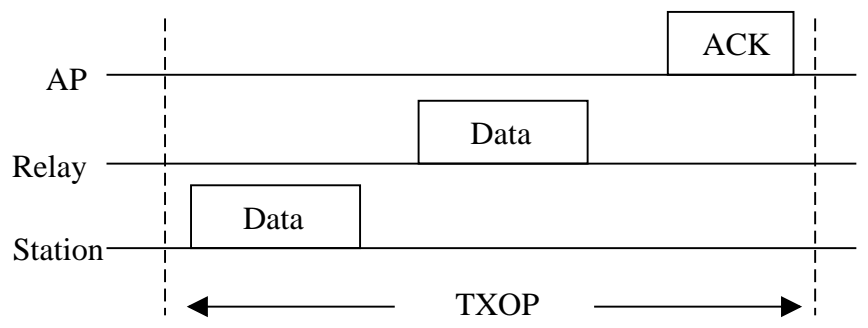

Fig. 4: TXOP operation for uplink video traffic with implicit ACK [15]

\section{Gauss-Markov Model for Drone Location Prediction:}

We deploy the mobility feature of drones in Gauss-Markov's model, which takes cognizance of transition from one state to another in three-dimension to predict the drone's movements. The Gauss-Markov Model considers the velocity of mobile drones tied with time and enables the drawbacks of realworld movements to be emulated. In this model, the velocity differences of drones are framed in a stochastic process and take advantage of the temporal dependencies feature, in addition to random properties. The current location value is 


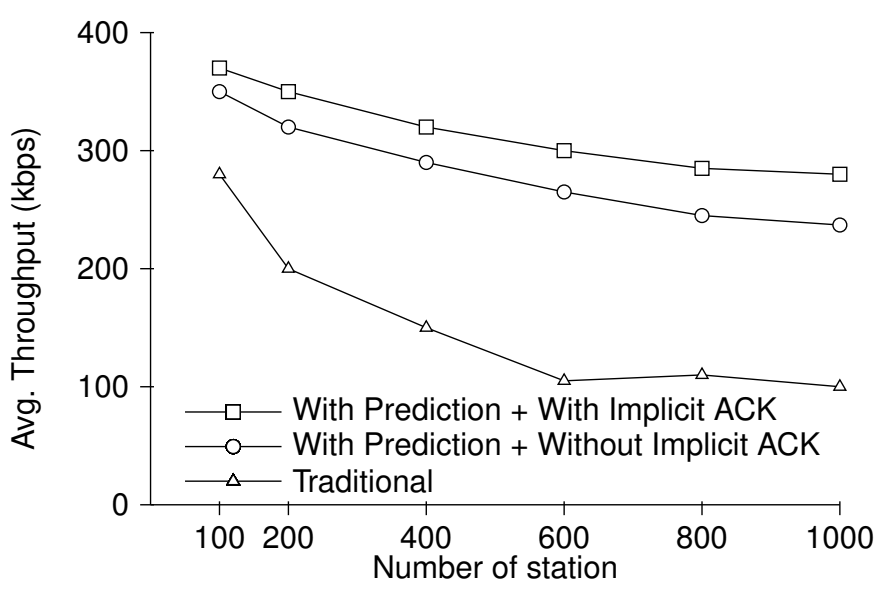

(a)

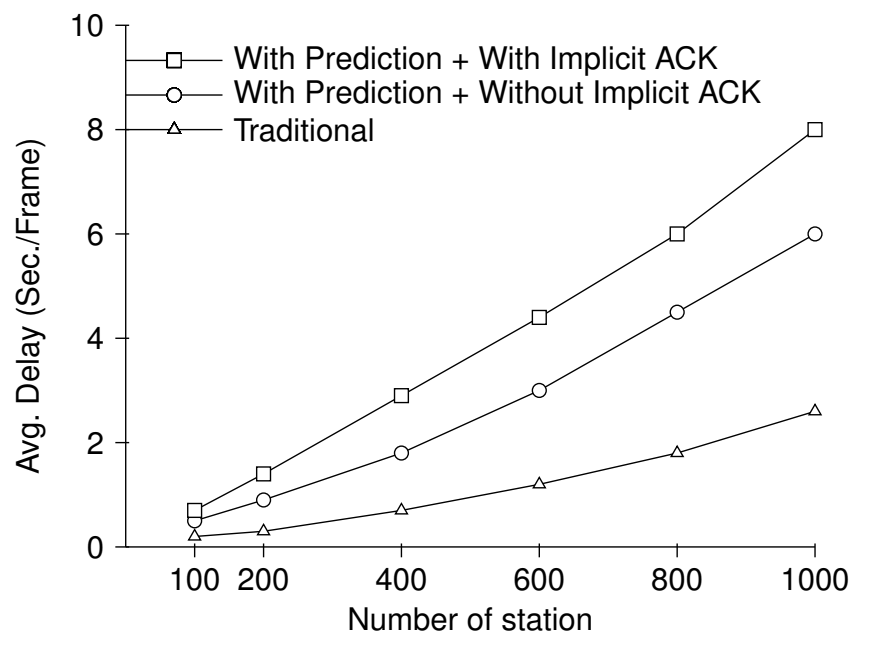

(b)

Fig. 5: (a) Average throughput performance as calculated at AP node, (b) Average delay incurs for different number of drone stations

within a specific range with a mean and standard deviation. A parameter called memory level i.e., $\alpha \in[0 ; 1]$ is considered in the Gauss-Markov model, which overrules the significance of the previous value while measuring the new value. The values are calculated mapping vector of trajectories into $\mathrm{X}, \mathrm{Y}$ and $\mathrm{Z}$ directions. In this model, using the set of historical data, the likelihood functions of each direction are calculated. In the Gauss-Markov model, we have the additional provision that can restrict the movements of the drones in the simulation. The direction and the velocity of the drones is changed after a specific time period $t$ by the controller. For $t$, the drone moves with static speed and direction. In the next step, when the drone finishes moving for $t$, based on the historical values and predefined values, it chooses a new value. If the drone moves towards the limit setup up in the simulation environment, the direction of the drone is altered 180 degrees that are to ask the drone from going beyond the boundary. If the drone moves towards an obstacle, the direction and pitches are shifted so that the drone stays in the designated area. This 3D trajectory gives a more realistic flavour than the random models in a mobile drone environment.

\section{B. Dynamic Traffic Scheduling}

The drones are grouped as per their current geographic location in the air. A RAW frame is allocated for the group of stations to continue their contention and transmission in a lot as per the mapping function of IEEE 802.11ah. As shown in Fig. 3, the drones are placed in the same RAW group with respect to their current location. If a drone's predicted location is found different location and under different relays, the AP sends the association/access configuration with a new relay node. In general, dynamic AID allocation is initiated by a nonAP node by sending an AID switch request. To enable this, the station is programmed with dot11DynamicAIDActivated equal to true. An AID switch frame is sent to a particular drone for using the new relay in our scheme.
In the considered drone network, the traffic moves from both upwards and downwards. However, stations send much higher volumes of traffic than receiving. The uplink traffic is the control data from AP to drones. Adopting the Implicit ACK procedure, effectively improves downlink traffic (i.e., drone stations to AP via relays) throughput. As shown in Fig. 4, we have three layers to downlink data packets. Firstly, in the implicit acknowledgement procedure, when a relay station receives a frame from a station associated with the relay, it does not send acknowledgement upon receiving. Rather, it forwards frames Short Interframe Space (SIFS) further. Secondly, when a station sends a frame to relay, it waits for a time-out and in this interval, upon receiving frames with the inclusion of Partial Association ID (PAID-hash of AID using Basic Service Set Identifier) in PHY header, it considers that the last sent frame was received successfully at the other end. And lastly, the AP collects all the PAID of stations coupled with the relay stations. The purpose of PAID is to secure data; on extracting the PAID in the PHY one station gets to know the destination station of the frame.

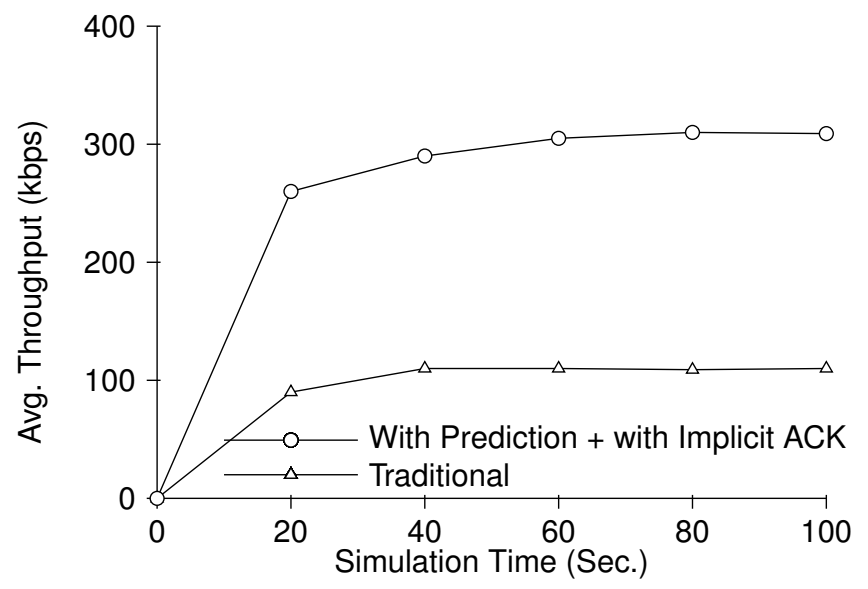

Fig. 6: Throughput performance as calculated at AP node 


\section{PERFormance EVAluation}

We analyze the proposed network architecture in Network Simulator-3 (NS-3). To simulate the mobility of the drones and relay drones, we use the Random Waypoint model. The selection of MCS is kept dynamic as per the data-rate requirements. MPEG-transport stream traffic is generated from the station towards the AP node. We analyze throughput performance, latency, and association with an increasing number of drone stations. We use Gauss-Markov Model for mobility simulation and prediction.

The proposed scheme with prediction and implicit ACK is compared with the traditional 802.11ah network. Fig. 5a shows throughput performance with an increasing number of mobile drone stations. The throughput in the traditional scheme decreases drastically due to the frequent associations and inefficient frame delivery through relay drones. As the proposed scheme predicts the future location, the required configuration related to channel access and association are dynamically sent to the respective relay drone. A slight lesser throughput is measured in case of not using the Block ACK mechanism. The proposed scheme increases throughput performance up to $58 \%$.

Similarly, the proposed scheme reduces contention and association delay significantly. As shown in Fig. 5b, the proposed scheme reduces up to $220 \%$ of average delay for 1000 stations. To see the association issues in the traditional scheme, we measure the average throughput with increasing simulation time (refer Fig. 6). The total stations (i.e., 1000) takes around $19 \mathrm{Sec}$. to be completely associated in the proposed network. Moreover, almost stable throughput performance can be seen throughout the simulation time. This is due to the dynamic association control by the AP node. Once a station gets associated with the AP, it does not need to do the same again in the network. AP manages the association by sending the association and access confirmation to the relay, which covers the position of the station. On the other hand, in the traditional scheme, every time a station needs to get associated with a new relay if it moves away.

\section{CONCLUSION}

This work presented an IEEE 802.11ah-based Internet of Drones architecture for surveillance and remote control. The proposed scheme considers the key challenges such as mobility of the drones and multi-hop coverage issues. Our solution predicts the location of a drone station and does (i) switching of stations modes and (ii) handover association and channel access configuration. The proposed scheme shows significant improvement in terms of throughput and latency.As part of one future work, we plan to analyse the proposed scheme with multiple MCSs, optimizing the size of TXOP, provisioning application-specific QoS requirements, handoff among APs, and finding a solution for better prediction accuracy.

\section{REFERENCES}

[1] L. Gupta, R. Jain, and G. Vaszkun, "Survey of important issues in UAV communication networks," IEEE Communications Surveys \& Tutorials, vol. 18, no. 2, pp. 1123-1152, 2015.
[2] G. A. Akpakwu, B. J. Silva, G. P. Hancke, and A. M. Abu-Mahfouz, "A survey on $5 \mathrm{G}$ networks for the Internet of Things: Communication technologies and challenges," IEEE access, vol. 6, pp. 3619-3647, 2017.

[3] N. Ahmed, H. Rahman, and M. I. Hussain, "An IEEE 802.11 ahbased scalable network architecture for Internet of Things," Annals of Telecommunications, vol. 73, no. 7, pp. 499-509, 2018.

[4] E. Kocan, B. Domazetovic, and M. Pejanovic-Djurisic, "Range extension in IEEE 802.11 ah systems through relaying," Wireless Personal Communications, vol. 97, no. 2, pp. 1889-1910, 2017.

[5] S. Kumar, H. Lim, and H. Kim, "Hierarchical MAC protocol with multichannel allocation for enhancing IEEE 802.11 ah relay networks," in 2015 International Wireless Communications and Mobile Computing Conference (IWCMC), pp. 1458-1463, IEEE, 2015.

[6] S. N. Rao, P. Akhil, V. B. Kumaravelu, and M. Arthi, "Dual - Hop Relaying for Quality of Service Improvement in IEEE 802.11ah Downlink," in 2018 International Conference on Communication and Signal Processing (ICCSP), pp. 0249-0253, 2018.

[7] N. Ahmed and M. I. Hussain, "Relay-based IEEE 802.11 ah network: A Smart City solution," in 2016 Cloudification of the Internet of Things (CIoT), pp. 1-6, IEEE, 2016.

[8] R. M. NA, D. Perdana, and R. M. Negara, "Performance analysis of mobility impact on ieee 802.11 ah standard with traffic pattern scheme," International Journal of Communication Networks and Information Security (IJCNIS), vol. 10, no. 1, 2018.

[9] A. A. Marwan, D. Perdana, and D. D. Sanjoyo, "Performance Analysis of RAW Impact on IEEE 802.11 ah Standard Affected by Doppler Effect," International Journal of Computers Communications \& Control, vol. 14, no. 2, pp. 212-219, 2019.

[10] M. Z. Ali, J. Misic, and V. B. Misic, "Extending the operational range of UAV communication network using IEEE 802.11 ah," in ICC 2019. 2019 IEEE International Conference on Communications (ICC), pp. 16, IEEE, 2019.

[11] A. Abdallah, M. Z. Ali, J. Mišić, and V. B. Mišić, "Efficient security scheme for disaster surveillance UAV communication networks," Information, vol. 10, no. 2, p. 43, 2019.

[12] S. Khan, M. Zeeshan, and Y. Ayaz, "Implementation and analysis of MultiCode MultiCarrier Code Division Multiple Access (MC-MC CDMA) in IEEE 802.11 ah for UAV Swarm communication," Physical Communication, vol. 42, p. 101159, 2020.

[13] L. Song, U. Deshpande, U. C. Kozat, D. Kotz, and R. Jain, "Predictability of WLAN mobility and its effects on bandwidth provisioning," 2006.

[14] X. Peng and Y. Zhang, "Location predicting methods for UAVs," in AIP Conference Proceedings, vol. 1864, p. 020109, AIP Publishing LLC, 2017.

[15] E. Khorov, A. Lyakhov, A. Krotov, and A. Guschin, "A survey on IEEE 802.11 ah: An enabling networking technology for smart cities," Computer communications, vol. 58, pp. 53-69, 2015. 